\title{
Small intestinal bacterial overgrowth in adult patients with type 1 diabetes
}

\author{
Małgorzata Bulanda*,Tomasz Gosiewski*, Monika Brzychczy-Włoch \\ Department of Microbiology, Jagiellonian University Medical College, Kraków, Poland
}

Correspondence to: Tomasz Gosiewski, PhD, Katedra Mikrobiologii, Uniwersytet Jagielloński, Collegium Medicum, ul. Czysta 18, 31-121 Kraków, Poland, phone: +48 126330877 , e-mail: tomasz.gosiewski@uj.edu.pl Received: August 29, 2016. Accepted: August 29, 2016. Conflict of interests: none declared. Pol Arch Med Wewn. 2016; 126 (9): 623-624 doi:10.20452/pamw.3574 Copyright by Medycyna Praktyczna, Kraków 2016

${ }^{*} \mathrm{MB}$ and $\mathrm{TG}$ contributed equally to this work.
In recent years, our understanding of the function of the human gastrointestinal tract has vastly improved, especially as regards the role of gastrointestinal microbiota (commensal intestinal flora). There is growing evidence for the significant effect of these microbes on the function and health of the human body. Commensal flora of the gastrointestinal tract plays a vital role in digestion and absorption of nutrients and protects against the invasion of pathogenic microorganisms by creating resistance to colonization and affecting the immune system of the host organism.

The gastrointestinal tract, especially in its final section, contains an enormous number of bacteria, which form a peculiar ecosystem. Gastrointestinal flora is an important part of the body. Its role and significance are increasingly recognized, which helps explain the course and causes of numerous diseases. An increasing number of studies have reported changes in microbiota composition in the course of various diseases, such as inflammatory bowel disease, ${ }^{1}$ celiac disease, ${ }^{2} \mathrm{di}-$ abetes, obesity, ${ }^{3}$ and many others.

Following this trend, Adamska et al, ${ }^{4}$ in the current issue of the Polish Archives of Internal Medicine (Pol Arch Med Wewn), presented the results of their studies on small intestinal bacterial overgrowth (SIBO) in patients with type 1 diabetes mellitus. The study included 148 adults with type 1 diabetes mellitus and 41 controls. SIBO was confirmed using a noninvasive hydrogen breath test performed with Gastro+Gastrolyzer (Bedfont Scientific Ltd., Maidstone, United Kingdom). The test consisted in assessing the concentration of exhaled hydrogen derived from 20 grams of lactulose administered orally and later decomposed by bacteria. The incidence of SIBO was shown to be significantly higher in the control group in comparison with the study group (73\% and $37.8 \%$, respectively, $P=$ 0.006 ), which probably came as surprise to the authors. ${ }^{4}$ Similarly, no significant correlations were demonstrated between the symptoms of SIBO and a positive result of the hydrogen breath test.
SIBO is defined as an overgrowth of bacterial flora inhabiting the small intestine and is usually described in association with irritable bowel syndrome. ${ }^{5}$ A number of studies reported the development of SIBO in patients who had undergone surgical treatment of gastrointestinal tumors [6] or other abnormalities in the gastrointestinal tract. ${ }^{7,8}$ In their paper, Adamska et $\mathrm{al}^{4}$ claimed that there had been no studies on the occurrence of SIBO in the course of diabetes, including type 1 diabetes. Indeed, there are few such studies, but isolated reports concerning type 1 and 2 diabetes are available. For example, Rana et $\mathrm{al}^{9}$ examined patients with type 2 diabetes and demonstrated a significant increase in the incidence of SIBO compared with the healthy population. Ojetti et $\mathrm{al}^{10}$ demonstrated that SIBO is present much more often in patients with type 1 diabetes who also have autonomic neuropathy, which is associated with a higher daily supply of insulin. Faria et al ${ }^{11}$ proved that intestinal transit can be inhibited in the course of type 1 diabetes, but the incidence of SIBO in this patient group is not significantly higher. This study was also cited by Adamska et al. ${ }^{4}$ It is therefore clear that the problem is not completely new, but it has not been well documented.

In their study, Adamska et $\mathrm{al}^{4}$ employed hydrogen breath testing to diagnose SIBO. The authors were right to point out that the gold standard for the diagnosis of SIBO is microbiological testing of a duodenal or jejunal aspirate; however, it is an invasive method. ${ }^{4}$ Breath testing enables a quick and noninvasive diagnosis of SIBO, but false results are possible (sensitivity of the test when lactulose is applied is $52 \%$, and specificity-86\%). ${ }^{12}$ Increased intestinal transit can reduce the sensitivity of the breath test, which causes a rapid removal of lactulose from the proximal small intestine. On the other hand, advanced lung diseases, such as tumors or inflammations, can result in false positive results due to the presence of abundant bacterial flora in the lungs. ${ }^{12}$ It seems 
that a similar misrepresentation of the breath test results may be caused by advanced tooth decay.

Clinical symptoms of SIBO are associated with bacterial overgrowth in the small intestine. Thus, antibiotic therapy is administered (eg, using rifaximin), which should reduce the number of bacterial cells and thus remove or alleviate symptoms. That is why, the use of antibiotic therapy should verify the results of the breath test. ${ }^{6}$ Long et $\mathrm{al}^{13}$ conducted a study with the use of breath testing and antibiotic therapy and demonstrated that the results did not coincide with a clinical improvement after the administration of rifaximin. For this reason, they questioned the significance of the breath test. It is a pity that the authors ${ }^{4}$ did not expand the study protocol to include the use of rifaximin or other antibiotic in patients in whom the breath test gave a positive result. Following antibiotic therapy, the test might have been repeated and the results juxtaposed with the patients' clinical state. Such an approach would have enhanced the value of the research. Surely, an invasive study involving the use of a duodenal aspirate and microbiological testing would be the best solution in this case, but it was not performed due to obvious reasons.

Adamska et $\mathrm{al}^{4}$ decided to apply a noninvasive method for diagnosing SIBO in patients with type 1 diabetes mellitus, which should be valued considering the scarce number of similar attempts. Our doubts concerning the applied method are not supposed to undermine the results of the analysis but rather to encourage the authors to conduct further research, which we hope they will undertake. It seems clear that the research on the impact of commensal microflora on the human body will continue to develop rapidly.

\section{REFERENCES}

1 Gosiewski T, Strus M, Fyderek K, et al. Horizontal distribution of the fecal microbiota in adolescents with inflammatory bowel disease. J Pediatr Gastroenterol Nutr. 2012; 54: 20-27.

2 Czaja-Bulsa G. Non coeliac gluten sensitivity - a new disease with gluten intolerance. Clin Nutr. 2015; 34: 189-194

3 Gosiewski T, Salamon D, Szopa M, et al. Quantitative evaluation of fungi of the genus Candida in the feces of adult patients with type 1 and 2 diabetes - a pilot study. Gut Pathog. 2014; 6: 43.

4 Adamska A, Nowak M, Pilacinski S, et al. Small intestinal bacterial overgrowth in adult patients with type 1 diabetes: its prevalence and relationship with metabolic control and the presence of chronic complications of the disease. Pol Arch Med Wewn. 2016; 126: 628-634.

5 Chu $H$, Fox $M$, Zheng $X$, et al. Small intestinal bacterial overgrowth in patients with irritable bowel syndrome: clinical characteristics, psychological factors, and peripheral cytokines. Gastroenterol Res Pract. 2016; 2016 3230859 .

6 Deng L, Liu Y, Zhang D, et al. Prevalence and treatment of small intestinal bacterial overgrowth in postoperative patients with colorectal cancer. Mol Clin Oncol. 2016; 4: 883-887.

7 Sung HJ, Paik CN, Chung WC, et al. Small intestinal bacterial overgrowth diagnosed by glucose hydrogen breath test in post-cholecystectomy patients. J Neurogastroenterol Motil. 2015; 21: 545-551.

8 Zhang $Y$, Feng $Y$, Cao B, et al. The effect of small intestinal bacterial overgrowth on minimal hepatic encephalopathy in patients with cirrhosis. Arch Med Sci. 2016; 12: 592-596

9 Rana S, Bhansali A, Bhadada S, et al. Orocecal transit time and small intestinal bacterial overgrowth in type 2 diabetes patients from North India. Diabetes Technol Ther. 2011; 13: 1115-1120.

10 Ojetti V, Pitocco D, Scarpellini E, et al. Small bowel bacterial overgrowth and type 1 diabetes. Eur Rev Med Pharmacol Sci. 2009; 13: 419-423.
11 Faria M, Pavin EJ, Parisi MC, et al. Delayed small intestinal transit in patients with long-standing type 1 diabetes mellitus: investigation of the relationships with clinical features, gastric emptying, psychological distress, and nutritional parameters. Diabetes Technol Ther. 2013; 15: 32-38.

12 Bures J, Cyrany J, Kohoutova D, et al. Small intestinal bacterial overgrowth syndrome. World J Gastroenterol. 2010; 16: 2978-2990.

13 Long SK, Di Palma JA. Does carbohydrate challenge testing predic clinical response in small intestinal bacterial overgrowth? South Med J. 2016; 109: 296-299. 